\title{
*INOCULATION OF MONKEYS WITH LEPROSY, FOLLOWING A DIET OF. PUAK (COLOCASIA)
}

\section{A Preliminary Report}

1). R. COLLIER

\section{INTRODUCTION}

A recent report by Cochrane(1) tells of his efforts in transmitting human leprosy to splenectomized monkeys. Adler (2) has reported the transmission of leprosy to Syrian hamsters, and Balfour-Jones ${ }^{(3)}$ the transmission of rat leprosy to the hamster. It is quite evident that, if there were a laboratory animal available for the study of leprosy, its mode of infection and various methods of treatment, we might expect much more rapid progress in the study of this disease, which has been so baffling for many hundreds of years. It is also self-evident that a method of infection, which follows actual conditions found in humans, is highly desirable. In the following experiments the animals were fed with a diet, which is common to a great many people throughout the world, as suggested by Oberdoerffer. ${ }^{(4)}$

Following Oberdoerffer's ideas on the role of adrenal insufficiency in the production of predisposition to leprosy, presumably caused by sapotoxins in certain food plants, Oberdoerffer and I thought it worth while to attempt to infect animals with leprosy, after a preliminary diet of puak. (Puak is the common Thai name for colocasia.) The following is a preliminary report on such an experiment :-

\section{Experimental Procedure}

Beginning September Ist, I938, two monkeys were put on a diet of colocasia. Since the animals refused to eat it raw it was prepared by boiling for a few minutes. Occasionally other articles of food such as bananas have been given, but on the whole the diet has been the one article of food-colocasia.

Since that time other monkeys have been added to our collection, so that we have now 30 animals under treatment. Guinea pigs, rabbits, and ordinary pigs have been used as well. I might add that we have obtained interesting results with the pigs. We are all aware of the very long incubation period of human leprosy. If leprosy in monkeys runs a parallel course, then it is probable that the incubation period will also be lengthy. Only a few of our monkeys have been under observation long enough to enable us

*Reprinted with the permission of the author from the Thai Science Bulletin, II, 2, April r940. 
to draw definite conclusions. It is probable that a prolonged period, possibly one or more years, will be necessary before final conclusions can be reached. However, in order that others interested in leper research may also attempt similar experiments, this preliminary report is being published.

Monkeys No. 1 and No. 2 were put on a diet of colocasia September Ist, 1938. The ordinary long tailed brown Rhesus monkey, native to North Thailand, was used throughout. On October Ist, a bit of tissue from an advanced lepromatous leper was inserted under the skin of the back and the opening closed with a skin-clip. On November 15th, some 6 weeks after inoculation, the female (No. I) developed an inflammation of the skin of the face, the palms of the hands, and the soles of the feet. The face was a deep red colour and somewhat swollen and infiltrated. The original lump at the site of inoculation on the back had more than doubled in size, but there was no sign of abcess formation. This "reaction" lasted about a month, and then subsided, though the thickening of the face below the eyes and across the eyebrows remained, and the face retained a reddish colour. A spot of depigmentation appeared on one side of the nose, which has remained ever since.

On March Ist, a skin scraping from the nodule of the back was found to contain acid-fast bacilli of the characteristic morphology of the mycobacterium leprae. On April I7th, I939, the animal was reinoculated in the same way as before. On May 2oth, and May 3oth, an ear clip was made and found to be negative. A nasal smear, made on the latter date, May 3oth, was positive for acidfast bacillae. Periodic examinations were made, and in every case the nasil smears were positive. Occasionally clumps of bacilli were

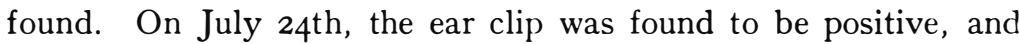
has remained so ever since. In October, a year after the first inoculation, a deep red colouration appeared across the buttocks, down the tail and across the back to the level of the lumbar vertebrae. There was a diffuse swelling of this area. Skin scrapings have been negative from this region. The face has at times been highly inflamed, but not at any time has it returned to norma' or lost its thickened reddish infiltration. The nipples have been red in colour and definitely hypertrophied. There has been no noticeable enlargement of the breast tissue. The ears have been enlarged and were red in colour.

The Male Monkey No. 2, inoculated at the same time as No. I and reinoculated similarly, has shown no signs of infection. His nasal smears and ear clips have been negative.

Monkey No. 3 (female) was inoculated December 15 th, and reinoculated April I7th, I939. On June 23rd, there was a positive 
nasal smear. On September 27 th, the nasal smear was positive, ear clip negative. From the first of September onward, the animal has had an intensely red induration of the face around the eyes, which has been persistent. This has not been noted in any of the control monkeys, nor in any monkeys which have not been treated at all. This has been accompanied with a swelling of the red area which shows in the illustration accompanying the article.

Monkey No. 4 Male. Inoculated with a culture made from leprous tissue. Leprous tissue was smeared on blood agar and after 24 hours the diphtheroid bacilli grown on this medium were injected in the form of a suspension. The only change following this injection was that the animal became very thin, though it remained as active as previously. On July $24^{\text {th }}$, a bit of leprous issue was rubbed inside of each nostril. There have been no symptoms of infection. Nasal smears since then have been negative.

Monkey No. 5 fiemale. Inoculated with a culture as described for monkey No. 4 on April I7th, 1939. Since there was no sign of infection, the animal was exposed by the rubbing of a nasal smear from monkey No. I in its nose. This was done July 24 th, and on August 26th, she had a positive nasal smear. There have been no skin changes. A nasal smear made October 15 th, was negative.

Monkey No. 6 Male. Injected with a culture as described above. There have been no further evidences of infection. Nasal smear negative on November Ist.

Monkey No. 7 Female. Inoculated with leprous tissue December I5th, and reinoculated April I 7 th. There were no skin changes, and on August 4th, she was again inoculated with leprous tissue. An abcess formed at the site of inoculation, and the micro-organisms found seemed to be entirely acid-fast bacilli. These were extremely numerous. No other micro-organisms were seen. This abcess healed spontaneously by the I3th (9 days later). On the 27 th of September a large mass was found on the opposite side of the back from the original inoculation. It was one inch long by three-fourths of an inch wide. It was soft and there was fluctuation. On aspirating the centre with a hypodermic needle, a purulent fluid was obtained which contained acid-fast bacilli. The edges of the mass remained indurated, and the mass has persisted to date. There were similar masses, though smaller in size on both wrists. There has been loss of hair on the back, and in the region of the tail. This monkey did not develop the facial changes of monkeys No. I and 3. The nasal smear has been positive. On November Ist, the ulnar nerves were found to be enlarged.

Monkey No. 11 was inoculated on August 5th, with leprous 
tissue. Beginning September I4th, he was given a number of injections of sapotoxin prepared from colocasia. This preparation was tested against rabbits, and $3 \mathrm{cc}$. of our solution killed a rabbit in 12 hours. The monkey was given $2 \mathrm{cc}$. of this preparation as an initial dose and the subsequent doses increased in amount. This was given in addition to the diet of colocasia, fed to the other experimental animals. By the first of October a mass was seen which grew slowly. On the first of November it had reached the size of a pigeon's egg-approximately one inch by three-fourths of an inch. This was situated some distance from the original inoculation, and on the opposite side of the back. The material aspirated from the centre contained acid-fast bacilli. Since then there has been a second mass located inferiorly to the first, and likewise it contained acid-fast bacilli. The eye-brows show thickening and are somewhat reddish in colour. The monkey has recently been seen to wipe his eyebrows repeatedly, sometimes continuing for as much as five minutes. We are inclined to believe that this may be due to paresthesia of the nerves around the eye. There has been loss of hair over the back and tail.

Monkeys 13, 14, 18, 19, were all inoculated with leprous material on August 4th. They have not been given colocasia. On December Ist, I939 none of them have shown any changes suggesting infection. The original lumps have disappeared. The nasal smears have been negative.

One baby monkey has been put into the cage with female No. 3 without being inoculated, but fed on colocasia, to see if contact of a baby with a positive foster mother monkey will result in infection. Observation will be continued in this case.

\section{Discussion of Results}

Our observations show that all four of the female monkeys inoculated six months or more ago have shown positive nasal smears and positive ear and skin clips. Only one male monkey has shown signs of infection. In that animal there have been abcesses, skin changes, and possibly paresthesia. That monkey has had injections of sapotoxins in addition to the diet of colocasia. The rate of progress has been much faster than with the other animals, and we are adopting this same technique with other monkeys in an attempt to speed up the period of incubation and the certainty of infection.

So far as we have been able to determine from the literature of the previous attempts to infect animals with leprosy there has been no mention of clinical tests such as ear clips, nasal smears, enlarged nerves etc. applied as a criterion of infection. We feel 
that such criteria are important since it is the clinical form of leprosy that is of value in such investigations. We believe that we have demonstrated such clinical signs in the experimental work covered in this preliminary report. The fact that several months elapse before symptoms appear is in accordance with our understanding of human leprosy. The clinical symptoms are very similar to those found in humans. The first positive symptom seems to be a positive nasal smear, though abcesses may occur previous to this. We have used this abcess material for inoculating other monkeys. The work has been done only recently and we must wait for a longer time before coming to conclusions. So far no control monkey has shown any of the symptoms noted for the inoculated animals who have been fed on colocasia.

The effect of colocasia on the infectivity of monkeys needs further study. It is suggested that a larger group be injected with sapotoxin from colocasia, in an effort to determine the exact role played by this food, and if possible to speed up the course of the disease and increase the certainty of infection. The juice from boiling colocasia appears to be very toxic to rabbits, and it may be that this will be effective in bringing about infection, following inoculation.

So far we have not attempted to treat any of our animals with anti-leprosy measures. This will be done later, as will pathological examination of internal organs. It is intended to report further progress with these experiments at a later date.

\section{Summary}

A number of monkeys have been fed on a diet of colocasia, and then inoculated with material from lepers. All four female monkeys, so treated six or more months ago, have developed symptoms similar to those seen in leprosy in humans. There have been positive bacteriological findings in nodules and changes in pigmentation of the skin. One animal developed thickening of the ulnar nerves. One male monkey has developed abcesses containing acid-fast bacilli as well as other symptoms after being injected with a solution of sapotoxin in addition to the diet of colocasia.

\section{References.}

( I) Cochrane, R. G. et. al. A preliminary note on inoculation of monkeys with human leprosy after splenectomy. Internat. Jl. Lep. VII, 3, p.377, July 1939.

(2) Adler. Inoculation of human leprosy in the Syrian hamster. Abstract in Internat. Jl. Lep. VI, 3, p.467, July 1938.

(3) Balfour Jones S. E. B. The experimental transmission of rat leprosy to the golden hamster. Jl. Path. and Bact. 45, 1937. 45 (1937).

(4) Oberdoerffer, M. J. Regional variation of clinical types in leprosy, seasonal variation of bacteriological findings in tuber- 
culoid leprosy, and their possible causation by sapotoxins in certain food plants. Congress of Far East Ass., Trop. Med. Nov. 1938.

IIn a later personal communication Dr. Collier says "I have taken a nasal smear from one monkey and rubled it in the nose of a second, which second has become positive. Also I have taken material from a nodule on one monkey and inoculated it into a second with positive results."

He also describes the method of extracting sapotoxin from colocasia:- "Peel the tubers, mince finely, mix with distilled water and leave for four hours. Add equal volume of $95 \%$ alcohol. Filter to remove precipitated starch; evaporate filtrate on water bath to remove alcohol. Add 30\% normal lead acetate. Filter-precipitate contains sapotoxin. Wash precipitate with distilled water. Add hydrogen sulphide, after having suspended precipitate in water; filter and evaporate filtrate to syrup. Add cold alcohol until white precipitate appears. Filter. White precipitate is very hygroscopic and contains inorganic impurities, but contains a large amount of sapotoxin. Dissolve in saline for injection. Determine minimum lethal dose for guinea pigs or rabbits by injection." Readers will find previous reference to colocasia in Lep. Review, X, 2, p.II2, July 1939.-Editor.] 